-31J. Inv. Ill-Posed Problems, Vol. 8, No. 1, pp. 23-30 (2000)

(c) VSP 2000

\title{
Theory of ground-penetrating radars III
}

\author{
A. G. RAMM*
}

Received June 22, 1999

\begin{abstract}
The governing equations are Maxwell's. The source of the current is either $j=e_{y} \delta\left(z-z_{0}\right) \delta(x) f(t)$ or $e_{\varphi} \delta\left(z-z_{0}\right) \delta\left(r-r_{0}\right) f(t)$ (straight wire or loop of current, $f(t)$ is a pulse). The conductivity $\sigma(z)$ and permittivity $\varepsilon(z)$ are unknown in the medium $(z>0)$ and are known in the air $(z<0)$. The source is in the air: $z_{0}<0$. The $\mu$ is a known constant. The data are the values of $E(x, y, z, t)$ and $H(x, y, z, t)$ for all $t>0$, all $x, y \in \mathbb{R}^{2}$ on the surface $z=0$. For $t<0$ the system is at rest: the electromagnetic fields vanish. Given the data, an exact inversion procedure is described to find $\sigma(z)$ and $\varepsilon(z)$.
\end{abstract}

\section{INTRODUCTION}

Consider the ground-penetrating radar problem (GPR):

$$
\nabla \times E=-\mu \frac{\partial H}{\partial t}, \quad \nabla \times H=\varepsilon(z) \frac{\partial E}{\partial t}+\sigma(z) E+j, \quad \text { in } \mathbb{R}^{3}
$$

$E=E(x, y, z, t), H=H(x, y, z, t)$ are electric and magnetic fields, the source

$$
j=e_{y} \delta\left(z-z_{0}\right) \delta(x) f(t)
$$

describes the straight wire with current along $y$-axis, $\delta(x)$ is the delta-function, $e_{y}$ is the unit vector along this axis, $z_{0}<0$ is the height at which the wire is located, $z>0$ is the medium (subsurface) region, $z<0$ is the region above the surface (the air, for example), $f(t)$ describes the shape of the time pulse, $\sigma(z)$ is the conductivity, $\varepsilon(z)$ is the dielectric permittivity, which are assumed known for $z<0$ and unknown for $z>0$. The function $f(t)$ is assumed to be compactly supported real-valued and bounded, for example, it can be a rectangular pulse or its smoothed version. The magnetic permittivity $\mu$ is a known constant, $\mu>0$. For $t<0$ the electromagnetic fields vanish.

\footnotetext{
* Mathematics Department, Kansas State University, Manhattan, KS 66506-2602, USA. E-mail: ramm@math.ksu.edu
} 
The data are the values

$$
E(x, y, t, z), \quad H(x, y, z, t), \quad \forall x, y \in \mathbb{R}^{2}, \quad \forall t>0 \quad \text { at } \quad z=0 .
$$

The GPR problem is: given the data (1.3), find $\varepsilon(z)$ and $\sigma(z)$ for $z>0$.

This problem has been studied in the literature, see $[5,6,9,10]$, and references therein. A method for analytical recovery of $\sigma(z)$ and $\varepsilon(z)$ from the data has been developed in [9]. In [6] a similar problem was solved for the case when the source was a loop of current

$$
j=e_{\varphi} \delta\left(z-z_{0}\right) \delta\left(r-r_{0}\right) f(t)
$$

where $r_{0}>0$ is the radius of the loop and $e_{\varphi}$ is the unit vector in the cylindrical coordinate system.

The analytical solution given in [6, 9], relies on the low-frequency exact inversion theory developed in $[3,5]$. The purpose of this paper is to develop an exact inversion theory for any frequency band.

\section{EXACT INVERSION THEORY FOR GRP PROBLEM}

The reduction of the GPR problem (1.1), (1.2) to an inverse problem for an ordinary differential equation (see equation (2.3) in [9])

$$
\begin{aligned}
u^{\prime \prime}-\lambda^{2} u+\omega^{2} A^{2}(z) u+\mathrm{i} \omega B(z) u & =-\delta\left(z-z_{0}\right), \quad z \in \mathbb{R} \\
u( \pm \infty, \lambda, \omega) & =0
\end{aligned}
$$

has been done in [9] for the source (1.2) and in [6] for the source (1.4). In equation (2.1) the source is defined by formula (1.2), the unknown function is $u=u(z, \lambda, \omega)$, the coefficients are:

$$
A^{2}(z):=\varepsilon(z) \mu, \quad B(z):=\sigma(z) \mu
$$

$\omega$ is the dual variable (in the Fourier transform) with respect to $t$, and $\lambda$ is the dual variable with respect to $x$. The data (1.3) yield the data

$$
u(0, \lambda, \omega) \quad \text { and } \quad u^{\prime}(0, \lambda, \omega), \quad \forall \lambda, \omega>0
$$

The prime denotes the derivative with respect to $z$. The vector-field $E(x, y, z, t)$ corresponding to the source (1.2) does not depend on $y$. Thus, it can be sought in the form $E(x, y, z, t)=\mathcal{E}(x, z, t) e_{y}$. The scalar function $\mathcal{E}(x, z, t)$ is related to $u(z, \lambda, \omega)$ by the formulas:

$$
u(z, \lambda, \omega):=[\mathrm{i} \omega \mu F(\omega)]^{-1} \int_{0}^{\infty} \mathrm{d} t \int_{-\infty}^{\infty} \mathrm{d} x \mathcal{E}(x, z, t) \mathrm{e}^{\mathrm{i} \omega t+\mathrm{i} \lambda x}
$$

and

$$
F(\omega):=\int_{0}^{\infty} f(t) \mathrm{e}^{\mathrm{i} \omega t} \mathrm{~d} t
$$


As we show below, the zeros of $F(\omega)$ in the region $\operatorname{Im} \omega>0$ are also zeros of the integral in $(2.5)$, so the function $u(z, \lambda, \omega)$ is well defined.

The zeros of $F(\omega)$ form a discrete set on the complex plane $\omega$ since $F(\omega)$ is an entire function of $\omega$ of exponential type. This is true because $f(t)$ is assumed compactly supported (it is a pulse in practice).

For rectangular pulse, for example, all zeros of $F(\omega)$ are real numbers (zeros of a sine-function), and the function $u(z, \lambda, \omega)$, defined in (2.5), which solves (2.1), is well defined at the zeros of $F(\omega)$ as the unique solution of (2.1) which vanishes as $|z| \rightarrow \infty$. In fact, the ratio (2.5) remains finite at all $\omega$ in the region $\operatorname{Im} \omega>0$, and at $\omega>0$.

Let us show that if $\omega$ in the region $\operatorname{Im} \omega>0$, is a zero of $F(\omega)$, then the integral in (2.5), which in this case solves the homogeneous problem (2.1), (2.2), must vanish.

Indeed, if $\omega=\omega_{1}+i \omega_{2}, \omega_{1} \neq 0, \omega_{2}>0$, then multiplying the homogeneous version of equation (2.1) by $\bar{u}$, (the overline stands for complex conjugate) integrating over $(-\infty, \infty)$, then by parts, and taking the imaginary part of the resulting expression, one gets:

$$
2 \mathrm{i} \omega_{1} \omega_{2}\left(A^{2} u, u\right)+\mathrm{i} \omega_{1}(B u, u)=0
$$

where $(u, v)$ is the scalar product in $L^{2}(\mathbb{R})$. Since $A^{2}(z)>0, B(z) \geq 0, \omega_{2}>0$, $\omega \neq 0$, it follows that $u \equiv 0$, as claimed.

Note also that equation (2.1) (with the requirement that its solution must vanish as $|z| \rightarrow \infty$ ) is equivalent to an integral equation which can be solved by iterations since it is an equation with the operator whose norm is small, so that the contraction mapping principle is applicable, for all sufficiently large $\lambda$ (see [6, equations (2.5) and (2.8)]), and the above integral equation has the unique solution.

The kernel of this integral equation depends analytically on $\omega>0$ (in [6] in equation (2.5) $k$ stands for $\omega$ ) and the equation is obviously uniquely solvable for sufficiently small $\omega$ for any fixed $\lambda$ in the half plane $\operatorname{Re} \lambda>0$ of the complex plane. If $A^{2}(z)$ and $B(z)$ are compactly supported or tend to zero as $|z| \rightarrow \infty$, then the integral operator in equation (2.5) in [6] is compact analytic operator function (in the space $\left.L^{2}(0, L)\right)$ of the parameter $\omega(k$ in $[6])$. Therefore, by the analytic Fredholm theorem (see [3, pp. 57-61]), the solution $u(z, \lambda, \omega)$, for any fixed $\lambda, \operatorname{Re} \lambda>0$, is analytic with respect to $\omega$ for all complex $\omega$ on the complex plane, except possibly for a discrete set. The function $u(z, \lambda, \omega)$ for any fixed $\omega$ is analytic with respect to $\lambda$ in the half-plane $\operatorname{Re} \lambda>c>0$, for sufficiently large $c>0$ (see equation (2.1)).

The function $\sigma(z)$ can be assumed compactly supported in many applications. In this case $B(z)$ is compactly supported. The function $A^{2}(z)$ is not compactly supported in some applications but can be assumed to take constant values at $+\infty$ and at $-\infty$, often different (see [9], for example).

In [9], equation (2.5), the integral operator for $u$ is linear, compact, and depends on the parameter $k$ ( $\omega$ in the present paper) analytically on the complex $\omega$-plane with cuts (see formula (2.8) in [9]). Since equation (2.5) in [9] is uniquely 
solvable for $\omega$ in $\operatorname{Im} \omega>0$, one concludes that $u(z, \lambda, \omega)$ is analytic in $\omega$ on the complex plane with cuts, except, possibly, for a discrete set of points.

The values (2.4) determine uniquely $u(0, \lambda, \omega)$ and $u^{\prime}(0, \lambda, \omega)$ in the regions

$$
\operatorname{Re} \lambda \geq 0, \quad \operatorname{Im} \omega \geq 0 \text {. }
$$

Let

$$
\begin{gathered}
\lambda=-\mathrm{i} k, \quad \omega=\mathrm{i} \nu, \quad \nu \geq 0, \quad \operatorname{Im} k \geq 0 \\
u(0,-\mathrm{i} k, \mathrm{i} \nu):=g(k, \nu), \quad u^{\prime}(0,-\mathrm{i} k, \mathrm{i} \nu):=h(k, \nu) \\
u(z,-\mathrm{i} k, \mathrm{i} \nu):=w(z, k, \nu) .
\end{gathered}
$$

Then

$$
\begin{gathered}
w^{\prime \prime}+k^{2} w-q(z, \nu) w=0 \quad \text { for } \quad z>0 \\
w(0, k, \nu)=g(k, \nu), \quad w^{\prime}(0, k, \nu)=h(k, \nu)
\end{gathered}
$$

$w$ is analytic in the region $\operatorname{Im} k>0, \quad \operatorname{Re} \nu>0$

$$
q(z, \nu):=\nu^{2} A^{2}(z)+\nu B(z)
$$

Define the impedance $I$-function (see [4] and [5, p. 288]):

$$
I(k):=\frac{h(k, \nu)}{g(k, \nu)}, \quad \forall k>0
$$

where $\nu>0$ is arbitrary fixed.

One can prove [8], that the $I$-function is identical to the Weyl function for potentials in the class $L_{1,1}$, which consists of real-valued potentials such that $x q(x) \in L^{1}\left(\mathbb{R}_{+}\right)$.

If $A^{2}(z)=A_{+}^{2}$ for $z>L$, where $A_{+}^{2}>0$ is a constant, and $L>0$ is an arbitrary large fixed number, then, assuming $B(z)=B_{+}=$const $\geq 0$ for $z>L$, denoting $\nu^{2} A_{+}^{2}+\nu B_{+}:=c_{+}^{2}$, where $c_{+}=$const $>0$, and defining $\overline{k^{2}}-c_{+}^{2}:=K^{2}$, $p(z, \nu):=q(z, \nu)-c_{+}^{2}$, one writes $(2.11)$ as

$$
w^{\prime \prime}+K^{2} w-p(z, \nu) w=0 \quad \text { for } \quad z>0
$$

where $p(z, \nu) \in L_{1,1}$.

Denote also $c_{-}^{2}:=\nu^{2} A_{-}^{2}+\nu B_{-}$, where $A_{-}^{2}$ and $B_{-}$are the constant values of the functions $A^{2}(z)$ and $B(z)$ for $z$ large negative. One has $I(K)=$ $I\left(\sqrt{k^{2}-c_{+}^{2}}\right), \operatorname{Im} \sqrt{k^{2}-c_{+}^{2}} \geq 0$. Therefore the values of $I(k)$ for $k>c_{+}$determine $I(K)$ for $K>0$, and the values of $I(k)$ for $k \in\left(0, c_{+}\right)$determine $I(K)$ for $K \in\left(0, i c_{+}\right)$. The function $I(K)$ is meromorphic in the region $\operatorname{Im} K>0$ with the only (simple) poles at the points $\mathrm{i} K_{j}, 1 \leq j \leq K$, where $-K_{j}^{2}$ are the bound states generated by the potential $p(z, \nu) \in L_{1,1}$. Therefore $I(K)$ is uniquely determined by its values on any open subset of $\mathbb{R}_{+}$.

It is proved in [4] (see also [5, pp. 288-291]) that $I$-function determines (for any fixed $\nu>0)$ the potential $p(z, \nu) \in L_{1,1}$ uniquely, and an algorithm is proposed for the recovery of $p(z, \nu)$ from $I(k)$. Necessary and sufficient conditions 
are given in [5] and [4] for a function $I(k)$ to be of the form (2.15), where $h$ and $g$ are defined by (2.12), $\nu$ is fixed, and $w$ solves $\left(2.11^{\prime}\right)$ with $p=p(z, \nu) \in L_{1,1}$. In [7], and especially in [8], one finds many applications of the $I$-function, its many properties and representation formulas [8], and an algorithms for finding the scattering data from the $I$-function.

Let us recall the recovery algorithm from [5, p. 288] and [4]. This algorithm allows one to recover the spectral function from the $I$-function and then the Gel'fand-Levitan inversion method applies. Another algorithm is developed recently in [8]. The algorithm from [8] allows one to recover the scattering data from the $I$-function and then the Marchenko inversion algorithm applies.

First, note that if $c_{+}=0$, that is, both functions $A^{2}(z)$ and $B(z)$ are compactly supported, then $k=K, q(z, \nu)=p(z, \nu) \geq 0$ and therefore there are no bound states of the operator $\mathrm{d}^{2} / \mathrm{d} z^{2}+q(z, \nu)$ in $L^{2}\left(\mathbb{R}_{+}\right)$(with the Dirichlet condition at $z=0)$. Under this assumption the spectral function $\mathrm{d} \rho(k)$ of this operator can be calculated by the formula:

$$
\mathrm{d} \rho(k)=\frac{2 k}{\pi} \operatorname{Im} I(k) \mathrm{d} k, \quad k>0 .
$$

Note that the solution $w$ of (2.11) is the solution proportional to the Jost solution $f(z, k)$ of (2.11), so that

$$
I(k)=\frac{f^{\prime}(0, k)}{f(k)}, \quad f(k):=f(0, k) .
$$

The Jost solution $f(z, k)$ is defined as the solution to (2.11) with the asymptotics

$$
f(z, k) \sim \mathrm{e}^{\mathrm{i} k z}, \quad z \rightarrow+\infty .
$$

One has $f(z, k)=\overline{f(z,-k)}$ for $k>0$,

$$
\operatorname{Im} I(k)=\frac{1}{2 \mathrm{i}}\left[\frac{f^{\prime}(0, k)}{f(k)}-\overline{\left(\frac{f^{\prime}(0, k)}{f(k)}\right)}\right]=\frac{k}{|f(k)|^{2}}
$$

where the Wronskian formula

$$
f^{\prime}(0, k) f(-k)-f^{\prime}(0,-k) f(k)=2 \mathrm{i} k
$$

was used. The spectral function is of the form:

$$
\mathrm{d} \rho=\frac{2 k^{2}}{\pi} \frac{\mathrm{d} k}{|f(k)|^{2}}
$$

and one gets (2.16) from (2.19) and (2.21).

The spectral function defines $q(z):=q(z, \nu)$ uniquely and algorithmically via the Gel'fand-Levitan procedure:

a) Since the non-negative potential (18) produces no bound state, one defines the kernel:

$$
L(z, y)=\int_{0}^{\infty} \frac{\sin (z k)}{k} \frac{\sin (y k)}{k} \mathrm{~d} \sigma(k), \quad \mathrm{d} \sigma:=\mathrm{d} \rho-\mathrm{d} \rho_{0}
$$


where

$$
\mathrm{d} \rho_{0}:=\frac{2 k^{2} \mathrm{~d} k}{\pi} .
$$

b) Then one solves the Gel'fand-Levitan integral equation for $K(z, y)$ :

$$
K(z, y)+L(z, y)+\int_{0}^{z} K(z, s) L(s, y) \mathrm{d} s=0, \quad 0 \leq y \leq z .
$$

Equation (2.24) is uniquely solvable: this is known (e.g., see [5, p. 261]).

c) If $K(z, y)$ is its solution, then

$$
q(z)=2 \frac{\mathrm{d} K(z, z)}{\mathrm{d} z}
$$

If $q(z)=q(z, \nu)$ is found for $\nu=\nu_{1}$ and $\nu=\nu_{2} \neq \nu_{1}$, then one finds $A^{2}(z)$ and $B(z)$ uniquely from the linear algebraic system:

$$
\nu_{1}^{2} A^{2}(z)+\nu_{1} B(z)=q\left(z, \nu_{1}\right), \quad \nu_{2}^{2} A^{2}(z)+\nu_{2} B(z)=q\left(z, \nu_{2}\right) .
$$

The determinant of this system is

$$
\nu_{1}^{2} \nu_{2}-\nu_{2}^{2} \nu_{1} \neq 0
$$

so system (2.26) is uniquely and analytically solvable for $A^{2}(z)$ and $B(z)$.

This completes the description of the exact inversion method for solving the GPR problem under the assumption that $A^{2}(z)$ and $B(z)$ vanish for $z>L$.

The more complicated case when $c_{+}>0$, so that bound states may occur, is discussed at the end of the paper.

Remark. One step of this method requires to find $u(0,-\mathrm{i} k, \mathrm{i} \nu), \nu>0$, $k>0$, from the given $u(0, \lambda, \omega)$ where $\lambda=-\mathrm{i} k, \omega=\mathrm{i} \nu$, which amounts to analytic continuation of the data from the real axis $\omega>0$ to the imaginary axis $\omega=\mathrm{i} \nu, \nu \geq 0$. This step can be done by the Cauchy formula

$$
u(0, \lambda, \mathrm{i} \nu)=\frac{1}{2 \pi \mathrm{i}} \int_{-\infty}^{\infty} \frac{u(0, \lambda, \omega)}{\omega-\mathrm{i} \nu} \mathrm{d} \omega, \quad \nu>0 .
$$

Recall that, for $\omega<0$, one has

$$
u(0, \lambda, \omega)=\overline{u(0, \lambda,-\omega)}
$$

so that the data $u(0, \lambda, \omega), \omega>0$, determines $u(0, \lambda, \omega)$ for $\omega<0$.

One also has to find $u(0,-\mathrm{i} k, \mathrm{i} \nu):=\varphi(k)$ from the knowledge of $u(0, \lambda, \mathrm{i} \nu):=$ $\psi(\lambda)$. The given function $\psi(\lambda)$ is analytic in the region $\operatorname{Re} \lambda>0$ and known for $\lambda>0$. This function can be written as

$$
\psi(\lambda)=\int_{0}^{\infty} \mathrm{e}^{-\lambda t} b(t) \mathrm{d} t
$$


Thus

$$
\varphi(k)=\int_{0}^{\infty} \mathrm{e}^{\mathrm{i} k t} b(t) \mathrm{d} t, \quad k>0 .
$$

Formulas (2.30) and (2.31) solve the problem of finding $u(0,-\mathrm{i} k, \mathrm{i} \nu)$ for $k>0$ from $u(0, \lambda, \mathrm{i} \nu)$ for $\lambda>0$.

The function $b(t)$ in (2.30) can be calculated from the data $\psi(\lambda), \lambda>0$, as the inverse Laplace transform. Inversion of the Laplace transform from the real axis is an ill-posed problem. An analytical formula for the Laplace transform inversion from the real axis is given in [5, p. 218]. There are some numerical methods for inversion of the Laplace transform from the real axis [1, 2].

Let us discuss now the case when $c_{+}>0$, so that the bound states may occur.

If the bound states $-K_{j}^{2}, 1 \leq j \leq J$, occur, then the Gel'fand-Levitan inversion procedure is still applicable but in formula (2.22) the integration should be taken from $-\infty$ to $+\infty$ with respect to $s:=k^{2}$, and $\mathrm{d} \rho(s):=$ $\sum_{j=0}^{J} c_{j} \delta\left(s+K_{j}^{2}\right) \mathrm{d} s$, where $\delta$ is the delta-function. Therefore one needs to find from the data (2.9) the values $K_{j}$ and $c_{j}$. The values $c_{j}$ are given by the formula $c_{j}=-2 \mathrm{i} K_{j} \operatorname{Res}_{K=\mathrm{i} K_{j}} I(K)$ (see [5, Section 8.4]). Thus, the residues of $I$-function at the points i $K_{j}$ determine $c_{j}$ explicitly.

Therefore one needs a method to find $K_{j}$ from the data. If all the bound states corresponding to the potential $p(z, \nu)$ lie on the segment $\left(-c_{+}^{2}, 0\right)$, then the numbers i $K_{j}$ can all be determined explicitly as the poles of $I(K)$ on the segment $\left(0, \mathrm{i} c_{+}\right)$of the imaginary axis of the complex $K$-plane.

This happens if $c_{-} \leq q(x, \nu)$, which is quite often the case in physics. If this assumption is not satisfied, then one has to determine the poles of $I(K)$ by analytic continuation from the real axis where $I(K)$ is known.

\section{REFERENCES}

1. B. Davies and B. Martin, Numerical inversion of the Laplace transform, J. Comp. Phys. (1978) 33, 1-32.

2. V. Krylov and N. Skoblya, Handbook of Numerical Inversion of Laplace Transform. Israel Progr. for Sci. transl., Jerusalem, 1969.

3. A. G. Ramm, Scattering by Obstacles. D. Reidel, Dordrecht, 1986.

4. A. G. Ramm, Recovery of the potential from $I$-function. Math. Reports of the Acad. of Sci., Canada (1987) 9, 177-182.

5. A. G. Ramm, Multidimensional Inverse Scattering Problems. Longman/Wiley, New York, 1992.

6. A. G. Ramm, Theory of ground-penetrating radars II. J. Inv. Ill-Posed Problems (1998) 6, No. 6, 515-520.

7. A. G. Ramm, Property C for ODE and applications to inverse scattering. Zeit. fuer Angew. Analysis (1999) 18, No. 2, 331-348. 
8. A. G. Ramm, Property C for ODE and applications to inverse problems. In the book: Operator Theory and its Applications, Amer. Math. Soc. and Fields Institute, providence RI, 2000 (Editors A.G.Ramm, P.N.Shivakumar, A.V.Strauss), pp. 1-62.

9. A. G. Ramm and A. Shcheprov, Theory of ground-penetrating radars. J. Inv. Ill-Posed Problems (1997) 5, No. 4, 377-384.

10. V.G. Romanov et al., An inverse problem for special dispersive media arising from ground-penetrating radar. J. Inv. Ill-Posed Problems (1997) 5, No. 2, 175-192. 